\title{
Preoperative Risk Factors for Predicting Missed Diagnosis of Renal Vein Tumor Thrombus in Renal Cell Carcinoma: A Retrospective Cohort Study
}

\section{Weixing Jiang}

University of the Chinese Academy of Sciences

Chuanzhen Cao

University of the Chinese Academy of Sciences

Hongzhe Shi ( $\nabla$ shihongzhe_cicams@163.com )

Cancer Hospital, Chinese Academy of Medical Sciences https://orcid.org/0000-0003-2276-9075

Jianzhong Shou

University of the Chinese Academy of Sciences

Dong Wang

University of the Chinese Academy of Sciences

Li Wen

University of the Chinese Academy of Sciences

Changling Li

University of the Chinese Academy of Sciences

Jianhui Ma

University of the Chinese Academy of Sciences

\section{Research article}

Keywords: Renal cell carcinoma; Renal vein tumor thrombus; Missed diagnosis

Posted Date: June 25th, 2019

DOI: https://doi.org/10.21203/rs.2.10631/v1

License: (1) (1) This work is licensed under a Creative Commons Attribution 4.0 International License.

Read Full License 


\section{Abstract}

Purpose: Previously, we have found some renal cell carcinoma (RCC) patients with renal vein tumor thrombus (RVTT) were misdiagnosed preoperatively. To improve the accuracy of diagnosis of RVTT in RCC, the clinical characteristics of RCC with missed diagnosis of RVTT were analyzed. Methods: We retrospectively reviewed RCC patients with RVTT between January 2000 and December 2015. Survival analysis was estimated using Kaplan-Meier. Cox proportional hazard models were applied to identify risk factors. Results: Missed diagnosis rate of RVTT in RCC was 30.5\%. In multivariate analysis, maximal tumor diameter $(O R=1.218, p=0.034)$ \tumor located in the middle part $(O R=1.354, p=0.003)$, renal vein contrast agents filling not well $(O R=1.252, p=0.015)$ and tumor with collateral vessels $(O R=1.218$, $\mathrm{p}=0.037)$ were independent predictors of missed diagnosis of RVTT. A missed-diagnosis score presented an AUC of 0.852 ( $p<0.001)$. In addition, missed diagnosis group patients had relative favorable prognosis, and tumor with collateral vessels was an independent prognostic indicator of poor OS of RVTT $(H R=1.153, p=0.025)$. Conclusions: To our knowledge, this was the first study that explored clinical features as predictors of missed diagnosis of RVTT. It should be alert to the possibility of complicating tumor thrombus at once with preoperative presence of renal tumor located in the middle part, renal tumor with collateral vessels, renal vein contrast agents filling not well.

\section{Background}

Renal cell carcinoma (RCC) accounts for approximately $2-3 \%$ of all malignant diseases in adults and has an increasing trend in china $[1,2]$. RCC has the tendency of invading blood vessels and the incidence to form venous tumor thrombus (VTT) is about $10 \%$ [3]. With the development of imaging in recent decades, there has been an increase in the detection of RCC involving VTT. Radical nephrectomy combining thrombectomy is the only curable method [4]. Different from the localized RCC, the long-term survival for patients with RCC involving tumor thrombus is relatively poor [5]. Once the VTT falls off, it will cause life-threatening pulmonary embolism. It is important to diagnose VTT in clinical practice.

The abdominal multi-parametric imaging, which can increase the discovery of low-stage renal masses, can identify the tumor thrombus in $4-10 \%$ of the patients [6]. CT, MRI and vascular ultrasonography are the main diagnostic methods for RCC with VTT. For Mayo grade I to IV of VTT, the rate of missed diagnosis is low. However, it is easy to miss VTT for Mayo 0 grade, the tumor thrombus only in the renal vein, which is the primary manifestation for renal vein invasion. There is currently no accurate preoperative clinical method to avoid misdiagnosis of the renal vein tumor thrombus (RVTT), if the renal vein is treated too close to RVTT intraoperatively, it is prone to cause RVTT to squeeze out and lead to pulmonary embolism.

Some RVTT have been misdiagnosed preoperatively in our institute [7]. In order to further analyze the causes of missed diagnosis of RVTT. We reviewed those RCC cases with RVTT in our hospital, in which the clinical and image characteristics were investigated. In addition, we also concluded predicting factors 
for helping diagnose RVTT with negative imaging diagnosis. These factors may have the ability to decrease the rate of misdiagnosis of RVTT.

\section{Methods}

\subsection{Study Population}

This was one of a series studies focused on RCC, which was approved by the Ethics Committee of National Cancer Center/Cancer Hospital, Chinese Academy of Medical Sciences (NCC/CHCAMS) (ID Num: NCC2016YJC-08). Patient consent was not required due to the retrospective study design. We focused on evaluating features of missed diagnosis of RVTT in RCC patients and found approaches to reduce the rate of missed diagnosis of RVTT.

We enrolled RCC patients with RVTT from the NCC/CHCAMS from January 2000 to December 2015. The medical records of each patients were retrospectively reviewed. All patients enrolled in the study met the 3-key inclusion criteria: $(I)$ patients underwent both enhanced abdominal CT and kidney MRI for clinical staging; (ii) patients were underwent nephrectomy with complete pathological specimens; (iii) histopathology-confirmation of RCC with RVTT (graded 0 tumor thrombus by Mayo classification). And then, patients meeting our inclusion criteria were classified and assigned to missed diagnosis group and diagnosis group according to whether the RVTT was misdiagnosed preoperatively, and sex and age matched patients followed 1:1 statistical matching were selected as control group (no tumor thrombus group), who were performed nephrectomy in the same continuous period.

\subsection{Preoperative assessment and surgical plan}

We collected image data including the tumor location, the maximal tumor diameter in the coronary plane, the filling circumstance of contrast agents, and the collateral vessels of tumor. Preoperative MRI and CT data were reviewed by two radiologists blind to patients' surgery information. Axial and coronal planes were combined to assess the status of contrast agents and collateral vessels. We defined poor filling that enhanced scanning of the renal vein phase (CT or MRI) appear any regional heterogeneous density (ref. Fig. 1). The criterion of collateral vessels around the tumor was at least one varicose vein around the tumor (ref. Fig. 1).

Lumbar or transabdominal radical nephrectomy were performed in all patients. After separating and clamping the renal artery during surgery, the upper and lower poles of the kidney were fully dissociated, and the renal vein was explored. If the tumor thrombus was found, the renal vein was clipped or ligated with Hem-o-lok at the beginning of the renal vein. This allowed for the complete removal of RVTT without tumor exposure.

\subsection{Statistical Analysis}

Normally distributed continuous data were compared using the Student's t-test, and the Chi-square test was used to compare the difference of distribution data between groups. Overall survival time (OS) were 
estimated by the Kaplan-Meier method and the Lon-rank test was used to compare different survival curves. Univariate and multivariate regression models were developed to find independent predictors for missed diagnosis of RVTT. The receiver operating characteristic curve (ROC) was constructed, and area under the curve (AUC) analysis was performed to determine prediction model. Statistical analysis was performed using IBM SPSS statistics for Windows, version 23.0 (IBM Corp. Armonk, NY, USA), and differences were considered statistically significant if $p$ values were $<0.05$.

\section{Results}

\subsection{Clinical characteristics of patients classified according to RVTT}

A total of 4426 RCC patients at NCC/CHCAMS were retrospectively reviewed. We screened out 128 (2.9\%) RCC patients according to inclusion criteria. The chief complaint was hematuria in $41.4 \%$ cases and $42.9 \%$ cases were diagnosed through routine physical examination. There were 103 males $(80.5 \%)$ and 25 females (19.5\%) in the 128 patients, with a median age of 61 years (range 34-87 years old). Among them, 39 patients $(30.5 \%)$ with RVTT misdiagnosed preoperatively by imaging were assigned to missed diagnosis group. 89 patients (69.5\%) diagnosed as RVTT through preoperative imaging and confirmed by postoperative pathology were assigned to diagnosis group. 39 patients followed 1:1 statistical matching with missed diagnosis group were assigned to no tumor thrombus group.

There were 22 cases $(56.4 \%)$ of tumors located in the middle of kidney in missed diagnosis group, 7 cases $(18.0 \%)$ of tumors in the upper renal pole, and 10 cases $(25.6 \%)$ in the lower renal pole. All clinical parameters were summarized in Table 1. No postoperative complications associated with embolism occurred. Missed diagnosis group was more likely to have bigger proportion of tumor located in the middle pole $(p=0.012)$, renal vein contrast agents filling not well $(p=0.032)$ and collateral vessels $(p=0.005)$ compared with no tumor thrombus group. These features have no difference between missed diagnosis group and diagnosis group.

\subsection{Development of the prediction model of missed diagnosis of RVTT}

Four preoperative variables including maximal tumor diameter [odds ratio (OR), 1.373; $95 \%$ confidence interval $(\mathrm{Cl}), 1.074-1.538 ; \mathrm{p}=0.015]$ ]tumor located in the middle part $(\mathrm{OR}, 1.892 ; 95 \% \mathrm{Cl}, 1.225-2.718$; $p<0.001)$, renal vein contrast agents filling not well $(O R, 1.397 ; 95 \% \mathrm{Cl}, 1.186-2.615 ; p<0.001)$ and tumor with collateral vessels $(\mathrm{OR}, 1.283 ; 95 \% \mathrm{Cl}, 1.013-1.864 ; \mathrm{p}=0.026)$ with $P$ values $<0.05$ in univariable analyses were considered as candidates for multivariable logistic regression analysis. They were significantly associated with missed diagnosis of RVTT, whereas BMI (Body Mass Index), KPS (Karnofsky Performance Status), paraneoplastic syndrome, and tumor side were not (Table 2).

Further multivariable logistic regression analysis demonstrated that maximal tumor diameter (OR, 1.218; $95 \% \mathrm{Cl}, 1.557-1.831 ; p=0.034)$ \tumor located in the middle part (OR, 1.354; $95 \% \mathrm{Cl}, 1.029-2.628 ; \mathrm{p}=0.003)$, renal vein contrast agents filling not well $(\mathrm{OR}, 1.252 ; 95 \% \mathrm{Cl}, 1.007-1.649 ; \mathrm{p}=0.015)$ and tumor with 
collateral vessels $(\mathrm{OR}, 1.218 ; 95 \% \mathrm{Cl}, 1.117-2.042 ; \mathrm{p}=0.037)$ were independent predictors of missed diagnosis of RVTT (Table 2).

On the basis of final multivariable model, a missed-diagnosis score was calculated by taking the sum of 1 score each for maximal tumor diameter, tumor located in the middle part, renal vein contrast agents filling not well, and tumor with collateral vessels (Fig. 2). The model presented an AUC of 0.852 (95\% Cl: 0.766-0.938, p<0.001) (Fig. 3).

\subsection{The correlation of clinical factors with outcome of different groups}

The median follow-up time was 47 months (7-186 months) of those patients. Missed diagnosis group achieved longer OS than diagnosis group and had similar survival time compared with no tumor thrombus group (Fig. 4).

Univariate and multivariate analyses for OS were summarized in Table 3 (among patients with RVTT). The univariate survival analysis revealed that tumor with collateral vessels was significantly associated with OS [HR (hazard ratio), 1.613; $95 \% \mathrm{Cl}, 1.328-2.715, \mathrm{p}<0.001]$. The maximal tumor diameter, tumor located in the middle side, and renal vein contrast agents filling not well were not significantly associated with OS. But the model that includes maximal tumor diameter, tumor located in the middle pole, renal vein contrast agents filling not well and tumor with collateral vessels, tumor with collateral vessels was an independent prognostic indicator of poor OS (HR, 1.153; 95\% Cl, 1.017-1.465, $\mathrm{p}=0.025)$.

\section{Discussion}

We have specially investigated Chinese RCC patients with RVTT and constructed a risk model for longterm survival in these patients [7]. On the basis of our report of high rate of missed diagnosis of RVTT, we further found that several features including maximal tumor diameter, tumor located in the middle part, renal vein contrast agents filling not well, and tumor with collateral vessels were associated with missed diagnosis of RVTT. These misdiagnosed patients had similar survival time with no tumor thrombus patients, which suggested that initially formed RVTT may be an unimportant prognosis factor.

According to the edition (8th) of the American Joint Committee on Cancer (AJCC) tumor-node-metastasis (TNM) staging system, RCC with VTT extending to the renal vein, extending to the sub diaphragmatic inferior vena cava, or extending to the supradiaphragmatic IVC (inferior vena cava) are classified as T3a, T3b, or T3c, respectively. VTT destroys the integrity of blood vessels and changes hemodynamics. Approximately $5 \%$ to $20 \%$ of RCC cases at the initial diagnosis are renal vein, inferior vena cava, or right atrial tumor thrombus. The probability of the VTT falling off and occurring embolism is between $1.2 \% \sim 6.0 \%$ [8]. The incidence rate is relatively low, while the mortality rate is high and there is no clinical evidence to support the implanted inferior vena cava filter can prevent pulmonary embolism $[9,10]$. RVTT belongs to T3a (locally advanced RCC) with the poor long-term survival. Radical nephrectomy combined with tumor thrombectomy is the only treatment option that may cure non-metastatic renal cell carcinoma with VTT [11-13]. However, 5 years cancer specific survival rate (CSS) was reported only $25 \%$ to $53 \%$ 
$[5,14,15]$. In our institute, the patients' CSS with RVTT was $67.9 \%$ in 5 years follow-up and $57.0 \%$ in 10 years follow-up [7]. In this study, we first investigated the prognosis of patients who were missed diagnosis of RVTT preoperatively. The result showed that these patients achieved longer survival than those have not been misdiagnosed RVTT, and also had similar survival time with no tumor thrombus patients. We considered that this kind of RVTT may be in a borderline status between obvious RVTT and initial formation of thrombus, which was needed to be paid special attention. More further studies were also expected.

RVTT is usually stable, but the risk of squeezing and embolization may occur in long, thin, or fragile RVTT tumor thrombus. Therefore, preoperative diagnosis of RVTT is particularly important. The diagnosis of RVTT mainly depends on CT, MRI, or vascular ultrasound, and the accuracy is approximately $85 \%$ [16-18]. The technical defects of imaging can contribute to missed diagnosis of RVTT. However, it also has a possible correlation with the characteristics of primary tumor. In this study, the average of tumor diameter in missed diagnosis group was $7.45 \mathrm{~cm}$. We found that there was no statistically significant difference in the maximum diameter of tumor between the missed diagnosis group and the no tumor thrombus group. However, maximal tumor diameter was an independent factor associated with missed diagnosis. In some degree, the diameter of tumor is relatively large in patients receiving radical nephrectomy which tends to compress the renal vein and disturb the imaging observation and judgment. Tumor size was reported of prognostic value and has not been specially investigated in RVTT $[19,20]$.

We also discovered that the proportion of tumors located in the middle part of kidney in missed diagnosis group was significantly higher than no tumor thrombus group. It may be that the middle kidney tumor is close to the renal pedicle vessels, it was difficult or unclear to identify the renal portal vessels from imaging due to the tumor inwardly growing, renal vein invasion, or tumor compression. There were also some RVTTs in renal vein phase of CT or MRI enhanced scanning only showed that insufficient contrast agent filling, which also sometimes could be found in normal renal vein because of contrast agents filling delay. The identification was also difficult, which still required combined other factors to provide assistance. Chopra S and his colleagues reported that a thorough understanding of collateral vessels was important for intraoperative safety of tumor thrombectomy for RCC [21]. Bradley AJ and his colleagues found that the presence of enlarged perinephric collateral vessels was more likely to have stage over T3a [22]. In this study, we discovered that patients with RVTT were prone to form collateral vessels, tumor with collateral vessels not only was a prognostic factor for RVTT, but also an important predictor of missed diagnosis of RVTT. In summary, according to literature, no other study evaluated the presence of these clinical features as predictors of missed diagnosis of RVTT.

However, the limitation of the present study was that it was a retrospective study including limited cases in certain groups. We will carry out prospective studies to confirm.

\section{Conclusions}


Missed diagnosis of RVTT may have relative favorable prognosis, and the feature of perinephric collateral vessels was a prognostic factor for RVTT. The imaging of RCC including the characteristics of large tumor diameter, tumor located in the middle part, renal vein contrast agents filling not well, and tumor with collateral vessels were associated with missed diagnosis of RVTT. These characteristics may not be found in all cases. But once one of the characteristics is recognized, it is necessary to have a high degree of suspicion of the possibility of combining RVTT.

\section{Declarations}

\section{Acknowledgements}

We specially thank Lianyu Zhang and Jin Zhang for reviewing preoperative MRI and CT data and preliminary establishment of the image database.

\section{Availability of data and materials}

The datasets used and/or analyzed data in the current study are available from the corresponding author on reasonable request.

\section{Funding}

This work was supported by the Chinese Academy of Medical Sciences (CAMS) Initiative for Innovative Medicine (Grant No. 2016-I2M-1-007) and Beijing hope run special fund of cancer foundation of China (LC2018L02).

\section{Ethics approval and consent to participate}

The research study was reviewed and approved by the Domain-Specific Review Board, Cancer Hospital Chinese Academy of Medical Sciences. Patient details were anonymized before analysis.

\section{Credit Authors statement}

Study design and revision of manuscript: Changling Li, Jianhui Ma

Data collection: Weixing Jiang, Chuanzhen Cao

Data analysis: Dong wang, Li Wen

Writing: Weixing Jiang

Critical revision of manuscript and final approval of the version to be submitted: Hongzhe Shi, Jianzhong Shou 
Statistical analysis: Weixing Jiang, Chuanzhen Cao

\section{Patient consent for publication}

Patient study consent was not required due to the study's retrospective nature.

\section{Competing interests}

The authors declare no conflicts of interests.

\section{References}

1. Siegel RL, Miller KD, Jemal A (2018) Cancer statistics, 2018. CA Cancer J Clin. 68:7-30.

2. Chen W, Zheng R, Baade PD et al (2016) Cancer statistics in China, 2015. CA Cancer J Clin. 66:115132.

3. Margulis V, Master VA, Cost NG et al (2011) International consultation on urologic diseases and the European Association of Urology international consultation on locally advanced renal cell carcinoma. Eur Urol. 60:673-683.

4. Wang B, Li H, Ma X et al (2016) Robot-assisted Laparoscopic Inferior Vena Cava Thrombectomy: Different Sides Require Different Techniques. Eur Urol. 69:1112-1119.

5. Tilki D, Nguyen HG, Dall'Era MA et al (2014) Impact of histologic subtype on cancer-specific survival in patients with renal cell carcinoma and tumor thrombus. Eur Urol. 66:577-583.

6. Abbasi A, Johnson TV, Ying K et al (2012). Duplicated vena cava with tumor thrombus from renal cancer: use of venogram for safer operative planning. Urology. 79: e57-58.

7. Cao C, Bi X, Liang J et al (2019) Long-term survival and prognostic factors for locally advanced renal cell carcinoma with renal vein tumor thrombus. BMC Cancer. 19:144.

8. Yokom DW, lhaddadene R, Moretto P et al (2014) Increased risk of preoperative venous thromboembolism in patients with renal cell carcinoma and tumor thrombus. J Thromb Haemost. 12:169-171.

9. Woodruff DY, Van Veldhuizen P, Muehlebach G et al (2013) The perioperative management of an inferior vena caval tumor thrombus in patients with renal cell carcinoma. Urol Oncol. 31:517-521.

10. Shah PH, Thompson RH, Boorjian SA et al (2018) Symptomatic Venous Thromboembolism is Associated with Inferior Survival among Patients Undergoing Nephrectomy with Inferior Vena Cava Tumor Thrombectomy for Renal Cell Carcinoma. J Urol. 200:520-527.

11. Gayed BA, Youssef R, Darwish 0 et al (2016) Multi-disciplinary surgical approach to the management of patients with renal cell carcinoma with venous tumor thrombus: 15 year experience and lessons learned. BMC Urol. 16:43.

12. Berczi A, Flasko T, Szerafin T et al (2017) Surgical Management and Outcome of Renal Cell Carcinoma with Inferior Vena Cava Tumor Thrombus. Urol Int. 99:267-271. 
13. Nini A, Capitanio U, Larcher A et al (2018) Perioperative and Oncologic Outcomes of Nephrectomy and Caval Thrombectomy Using Extracorporeal Circulation and Deep Hypothermic Circulatory Arrest for Renal Cell Carcinoma Invading the Supradiaphragmatic Inferior Vena Cava and/or Right Atrium. Eur Urol. 73:793-799.

14. Sidana A, Goyal J, Aggarwal P, Verma P, Rodriguez R (2012) Determinants of outcomes after resection of renal cell carcinoma with venous invlvement. Int Urol Nephrol. 44:1671-1679.

15. Kaushik D, Linder BJ, Thompson RH et al (2013) The impact of histology on clinicopathologic outcomes for patients with renal cell carcinoma and venous tumor thrombus: a matched cohort analysis. Urology. 82:136-141.

16. Gupta NP, Ansari MS, Khaitan A et al (2004) Impact of imaging and thrombus level in management of renal cell carcinoma extending to veins. Urol Int. 72:129-134.

17. Hallscheidt PJ, Fink C, Haferkamp A et al (2005) Preoperative staging of renal cell carcinoma with inferior vena cava thrombus using multidetector $\mathrm{CT}$ and MRI: prospective study with histopathological correlation. J Comput Assist Tomogr. 29:64-68.

18. Zhang LJ, Wu X, Yang GF et al (2013) Three-dimensional contrast-enhanced magnetic resonance venography for detection of renal vein thrombosis: comparison with multidetector CT venography. Acta Radiol. 54:1125-1131.

19. Schiavina R, Borghesi M, Chessa F et al (2015) The Prognostic Impact of Tumor Size on CancerSpecific and Overall Survival Among Patients With Pathologic T3a Renal Cell Carcinoma. Clin Genitourin Cancer. 13:e235-e241.

20. Brookman-May SD, May M, Wolff I et al (2015) Evaluation of the prognostic significance of perirenal fat invasion and tumor size in patients with pT1-pT3a localized renal cell carcinoma in a comprehensive multicenter study of the CORONA project. Can we improve prognostic discrimination for patients with stage pT3a tumors? Eur Urol. 67:943-951.

21. Chopra S, Simone G, Metcalfe C et al (2017). Robot-assisted Level II-III Inferior Vena Cava Tumor Thrombectomy: Step-by-Step Technique and 1-Year Outcomes. Eur Urol. 72:267-274.

22. Bradley AJ, MacDonald L, Whiteside S, Johnson RJ, Ramani VA (2015) Accuracy of preoperative CT T staging of renal cell carcinoma: which features predict advanced stage? Clin Radiol. 70:822-829.

\section{Tables}

Table 1. Clinical characteristics between different groups 


\begin{tabular}{|c|c|c|c|c|c|}
\hline Characteristics & $\begin{array}{l}\text { Missed diagnosis } \\
\text { group }\end{array}$ & $\begin{array}{c}\text { No tumor thrombus } \\
\text { group }\end{array}$ & $\begin{array}{l}\text { Diagnosis } \\
\text { group }\end{array}$ & $\begin{array}{c}P_{1} \\
\text { value* }\end{array}$ & $\begin{array}{l}P_{2} \\
\text { value }^{*}\end{array}$ \\
\hline \multicolumn{6}{|l|}{ MEAN (SD) } \\
\hline BMI, $\mathrm{kg} / \mathrm{m}^{2}$ & $26.74(3.12)$ & $23.68(3.72)$ & $25.12(1.93)$ & 0.172 & 0.677 \\
\hline Tumor diameter, $\mathrm{cm}$ & $7.45(3.28)$ & $6.03(3.74)$ & $6.53(1.87)$ & 0.552 & 1.658 \\
\hline \multicolumn{6}{|l|}{$\mathbf{N}(\%)$} \\
\hline KPS & & & & 0.556 & 0.911 \\
\hline$\square 80$ & $2 \square 5.1 \square$ & $1 \square 2.6 \square$ & $5 \square 5.6 \square$ & & \\
\hline$\geq 80$ & $37 \square 94.9 \square$ & $38 \square 97.4 \square$ & $84 \square 94.4 \square$ & & \\
\hline Side & & & & 0.365 & 0.399 \\
\hline Left kidney & $17(43.6)$ & $21(53.8)$ & $46(51.7)$ & & \\
\hline Right kidney & $22(56.4)$ & $18(46.2)$ & $43(48.3)$ & & \\
\hline Tumor location & & & & 0.012 & 0.056 \\
\hline Middle & $22(56.4)$ & $11(28.2)$ & $34(38.2)$ & & \\
\hline Upper and lower & $17(43.6)$ & $28(71.8)$ & $55(61.8)$ & & \\
\hline Paraneoplastic syndrome & & & & 0.556 & 0.452 \\
\hline Yes & $1(2.6)$ & $2(5.1)$ & $5(7.9)$ & & \\
\hline No & $38(97.4)$ & $37(94.9)$ & $84(92.1)$ & & \\
\hline Renal vein contrast agents filling & & & & 0.032 & 0.860 \\
\hline \multicolumn{6}{|l|}{ not well } \\
\hline Yes & $18(46.2)$ & $9(23.1)$ & $38(42.7)$ & & \\
\hline No & $21(53.8)$ & $30(76.9)$ & $51(57.3)$ & & \\
\hline Tumor with collateral vessels & & & & 0.005 & 0.834 \\
\hline Yes & $13(33.3)$ & $3(7.7)$ & $28(31.5)$ & & \\
\hline No & $26(66.7)$ & 36 (92.3) & $61 \quad(68.5)$ & & \\
\hline
\end{tabular}

* $P_{1}$, Comparison between missed diagnosis group and no tumor thrombus group; $P_{2}$, Comparison between missed diagnosis group and diagnosis group 
Table 2. Univariate and multivariate COX analysis for clinical factors in predicting missed diagnosis of RVTT

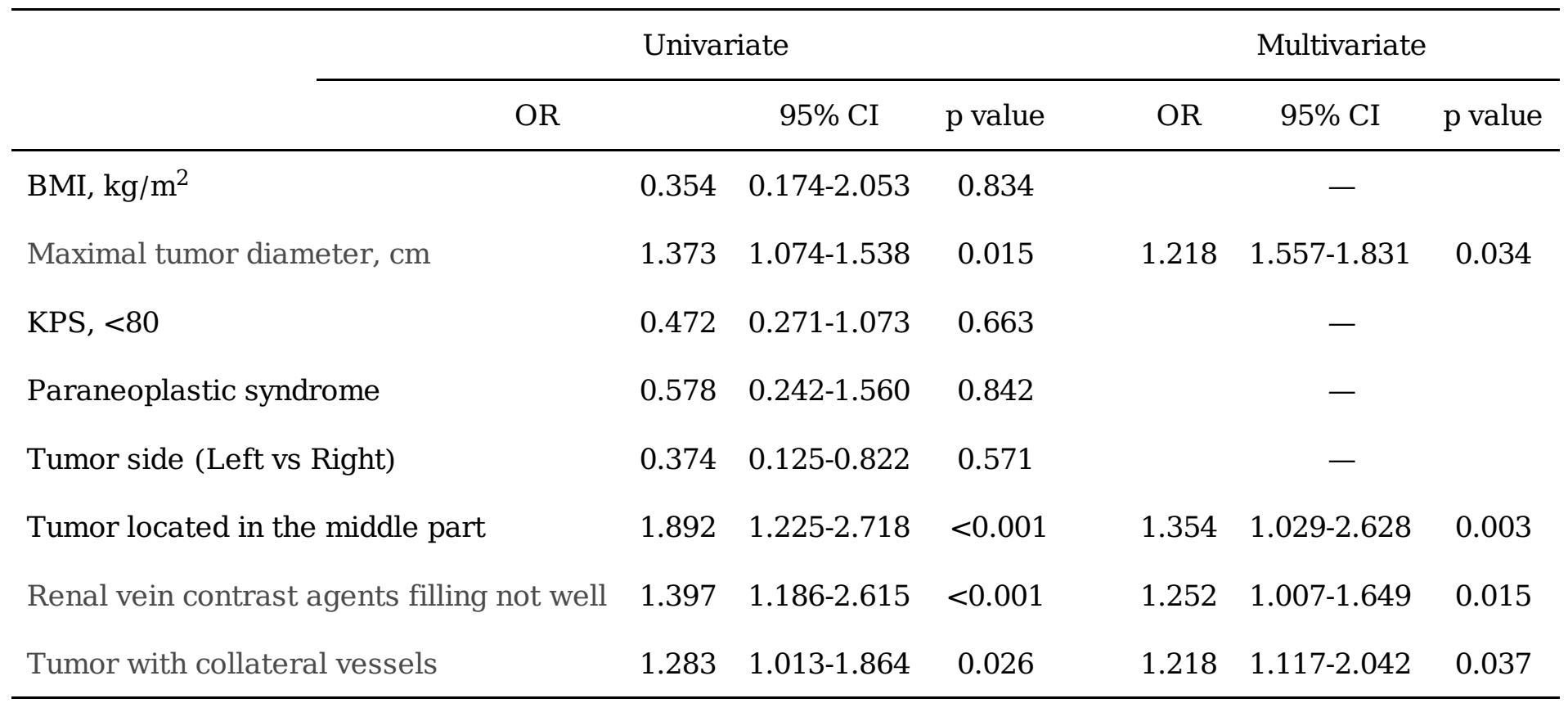

Table 3. Univariable and multivariable COX analysis for clinical factors in predicting prognosis of RVTT

\begin{tabular}{lccccccc}
\hline & \multicolumn{3}{c}{ Univariate } & & \multicolumn{3}{c}{ Multivariate } \\
\cline { 2 - 8 } & HR & & 95\% CI & p value & HR & 95\% CI & p value \\
\hline Maximal tumor diameter, cm & 0.612 & $0.114-1.057$ & 0.748 & 0.873 & $0.162-1.718$ & 0.917 \\
Tumor located in the middle part & 0.249 & $0.105-0.812$ & 0.569 & 0.648 & $0.059-1.221$ & 0.514 \\
Renal vein contrast agents filling not well & 0.176 & $0.088-0.569$ & 0.406 & 0.526 & $0.251-1.136$ & 0.835 \\
Tumor with collateral vessels & 1.613 & $1.328-2.715$ & $<0.001$ & 1.153 & $1.017-1.465$ & 0.025 \\
\hline
\end{tabular}

\section{Figures}




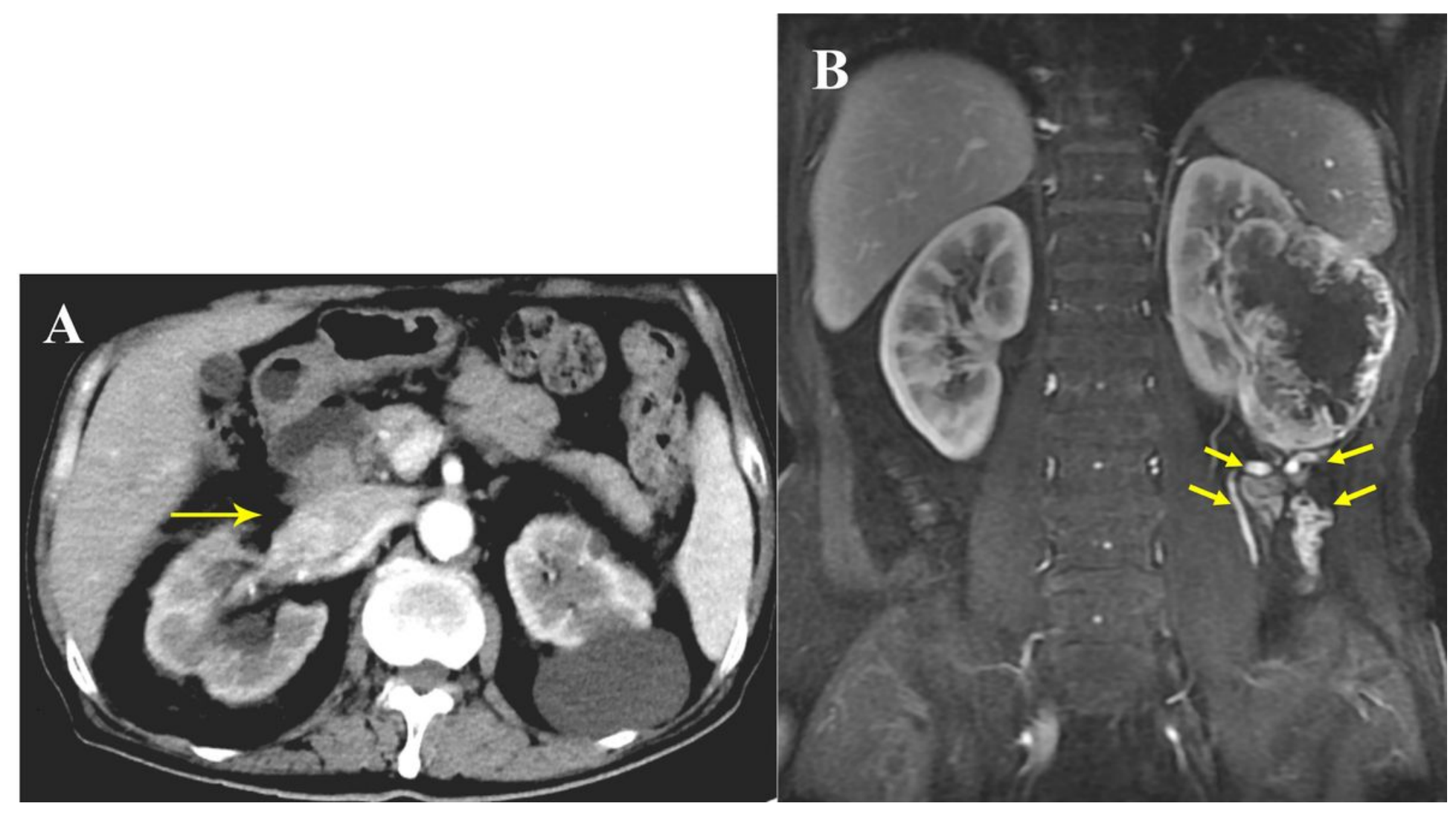

Figure 1

Appraisal of renal vein contrast agents filling not well and tumor with collateral vessels. (A) CT imaging of poor contrast agent filling (arrow) in the renal vein; (B) MRI imaging of renal tumor with enlarged collateral vessels. 
Achieved scores in missed diagnosis group

18

16

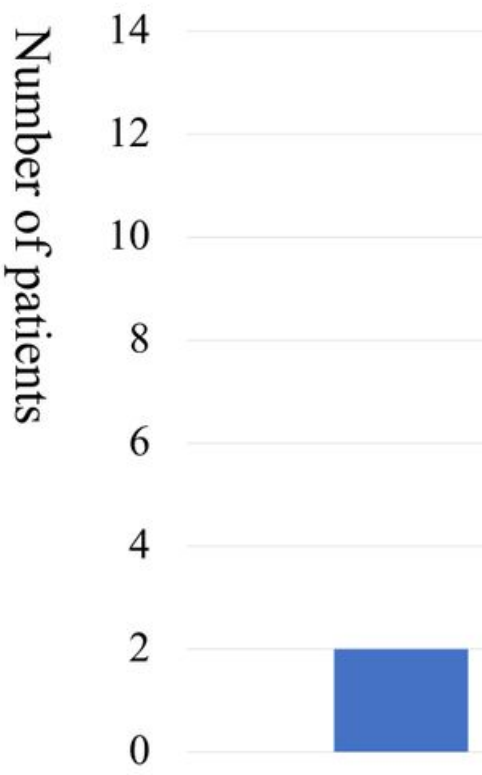

One score

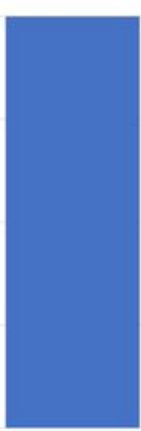

Two scores

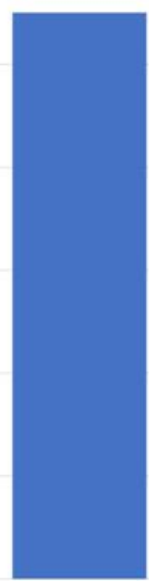

Three scores

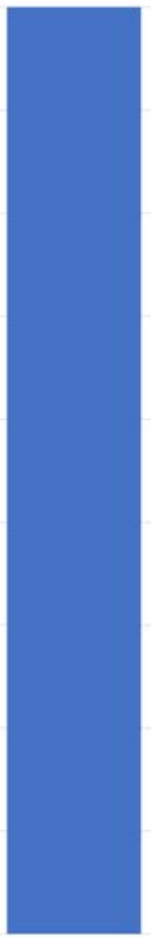

Four scores

\section{Figure 2}

The sum scores for maximal tumor diameter, tumor located in the middle part, renal vein contrast agents filling not well and tumor with collateral vessels (one score for each feature). 


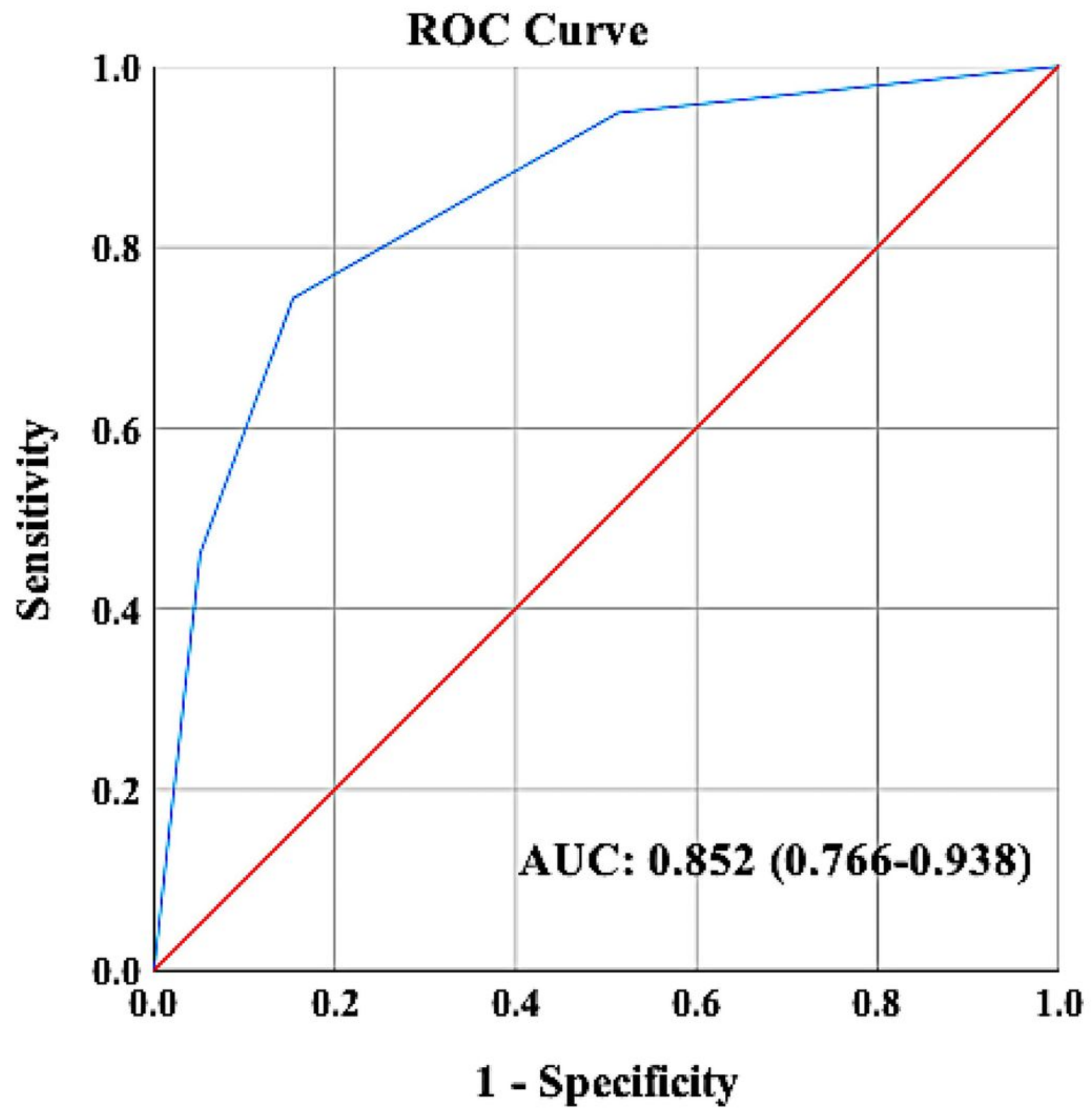

Figure 3

Scores based on final multivariable model were assessed by ROC curve. 


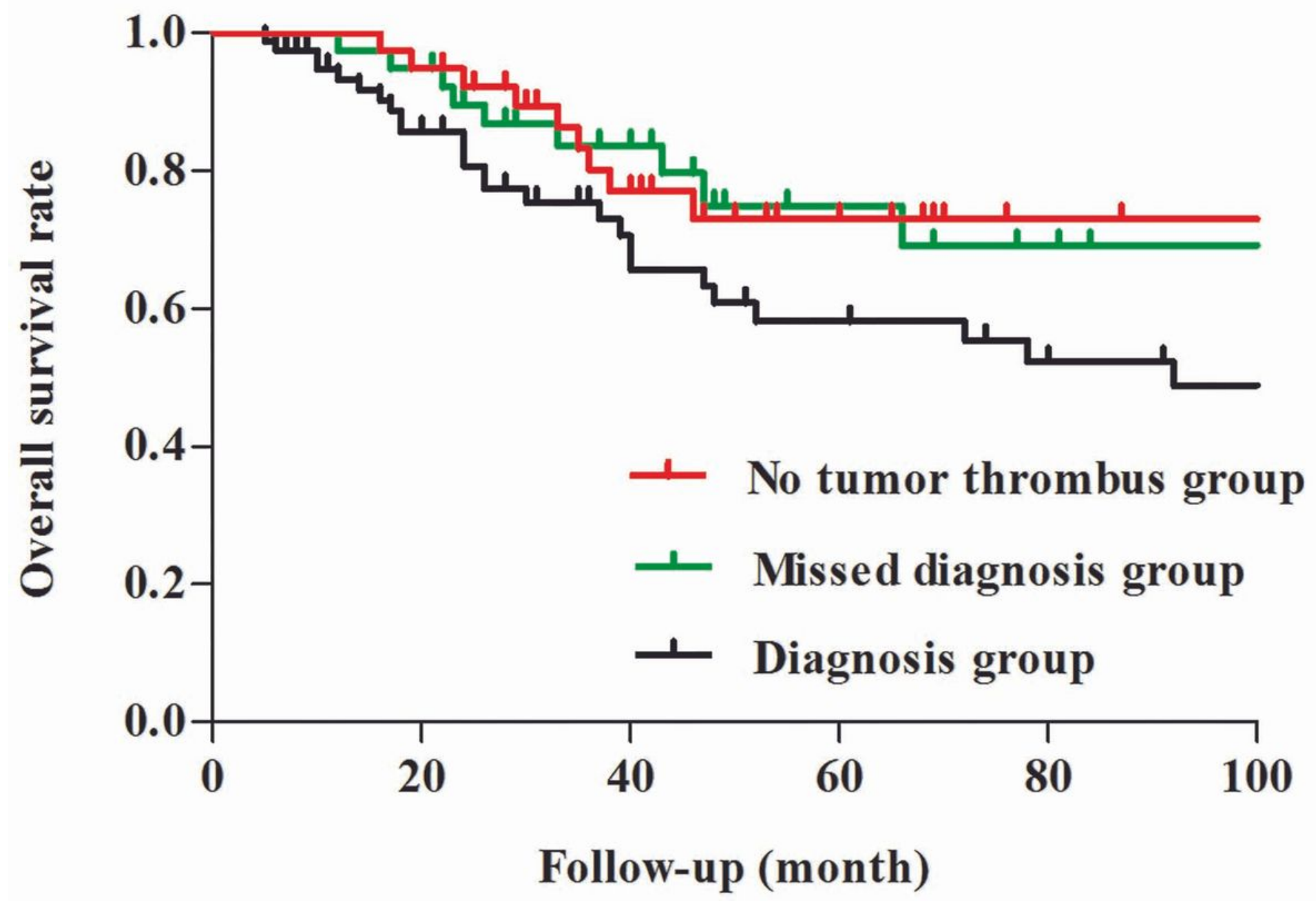

Figure 4

The correlation of overall survival between three groups. Diagnosis group patients had poorer survival time than missed diagnosis group and no tumor thrombus group ( $p=0.041$ and $p=0.033$ respectively). 